$$
\begin{aligned}
& E_{010} \text {-Schwingung: } c=\begin{array}{l}
299793 \pm 6 \mathrm{~km} / \mathrm{sec} \\
299796 \pm 6 \mathrm{~km} / \mathrm{sec}
\end{array} \\
& E_{011} \text {-Schwingung: } c=\begin{array}{l}
299792 \pm 9 \mathrm{~km} / \mathrm{sec} \\
299790 \pm 9 \mathrm{~km} / \mathrm{sec}
\end{array}
\end{aligned}
$$

Bildet man daraus einen gewogenen Mittelwert, so ergibt sich als wahrscheinlichster Wert für die Licht- geschwindigkeit:

$$
c=299793 \mathrm{~km} / \mathrm{sec}
$$

mit einem maximalen Fehler von $\pm 6 \mathrm{~km} / \mathrm{sec}$ gegenüber dem von L. Essen und G.Smith gefundenen Wert von: $c=299792 \pm 9 \mathrm{~km} / \mathrm{sec}$.

\title{
Über die Ausbreitung elektromagnetischer Wellen in einem atmosphärischen Wellenleiter
}

\author{
Von Th. Kahan und G. Eckart" \\ (Z. Naturforschg. 5 a, 334-342 [1950]; eingegangen am 23. März 1950)
}

\begin{abstract}
An Hand eines einfachen Modells wird die Ausbreitung elektromagnetischer Wellen in einem atmosphärischen Weilenleiter („Duct“ der englischen und amerikanischen Literatur) untersucht und besonders das Auftreten von Hohlleiterwellen studiert. Wir sind zu folgenden Resultaten gekommen:

a) Oberhalb des Wellenleiters unter einigermaßen steilen Erhebungswinkeln hat man eine Ausbreitung nach dem Gesetz $1 / R$, das mit $(z-h)$ gegen Null geht.

b) In der Zone unmittelbar über der Trennebene nimmt das Feld exponentiell mit der Höhe ab.

c) Diese beiden Erscheinungen beschreiben den Effekt der toten Zone.

d) Im Wellenleiter haben wir asymptotisch:

a) Wenn er niedriger ist, als einer bestimmten Höhe entspricht (oder wenn die Frequenz niedriger ist als eine bestimmte Grenzfrequenz), ein Gesetz $1 / r^{3}$ für die Feldstärken.

$\beta$ ) Wenn der Wellenleiter oder die Frequenz höher ist, Hchlleiterwellen der Ordnung $1 / V r$.

e) In dem Zwischengebiet bis zum Auftreten der Totalreflexion hat man für das Feld ein Gesetz der Ordnung $1 / r$, das man durch sukzessive Reflexionen beschreiben kann.

f) Damit ist zugleich die Theorie einer unendlich ausgedehnten dielektrischen Flächenantenne gegeben.
\end{abstract}

$\mathrm{W}$ ir setzen eine unendlich leitende ebene Erde voraus. Darüber befinde sich ein Luftraum der Dielektrizitätskonstanten $\varepsilon_{1}$. In der Höhe $h$ vermindere sich diese Dielektrizitätskonstante unstetig auf $\varepsilon_{\mathbf{2}}$. In der Höhe $\zeta$ über dem Erdboden befinde sich ein magnetischer Vertikaldipol, von dem ein Feld ausgestrahlt wird, das sich aus den Komponenten $E_{\varphi}$, $H_{r}, H_{z}$ zusammensetzt, wenn $r, \varphi, z$ Zylinderkoordinaten bedeuten ${ }^{1,2,5}$ (Abb. 1 u. 2). Dieser Sendertyp wurde vor allem aus folgendem Grund gewähli: Über unendlich leitendem Erdreich bildet sich ein

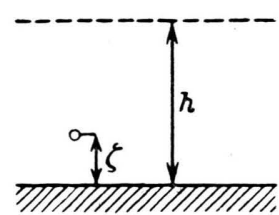

Abb. 1.

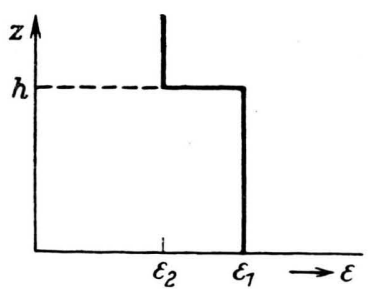

Abb. 2
Abb. 1. Die räumliche Anordnung des Problems. Abb. 2. Die Verteilung von $\varepsilon$.
Vertikaldiagramm des Senders aus, das dem über endlich leitendem Boden der Größenordnung $1 / R$ gleicht; in der Ordnung $1 / R$ zieht dieses nämlich auf Null ein. Ein vertikaler elektrischer Dipol würde am Boden stets das Feld der Abrahamschen Lösung ergeben, wenn man die Leitfähigkeit unendlich hoch annimmt. Bei jeder endlichen Leitfähigkeit aber müßte sein Feld in der Ordnung $1 / R$ am Boden auf Null einziehen. Wir werden also unter Voraussetzung unendlicher Leitfähigkeit den wirklichen Verhältnissen mit einem magnetischen Dipol (horizontaler $\varepsilon$-Polarisation) viel eher gerecht als mit einem elektrischen Vertikaldipol.

\section{Mathematischer Teil}

1. Aufstellung der Differentialgleichung und der Grenzbedingungen

Unter Verwendung praktischer (MKS)-Einheiten, der Zeitfunktion $e^{-i \omega t}$ und eines Zylinderkoordinatensystems $r, \varphi, z$ definieren wir einen Fitzgerald-

* Neuilly-sur-Seine, 4 Villa du Roule. 
schen· Vektor analog zum Hertzschen Vektor durch

$$
E=-i \omega \operatorname{rot} \Pi \text {. }
$$

Dann gilt für $\Pi$ die Wellengleichung:

wo

$$
\begin{gathered}
\Delta \Pi_{1,2}+k_{1,2}^{2} \Pi_{1,2}=0, \\
k_{1,2}^{2}=\omega^{2} \mu_{0} \mu \varepsilon_{0} \varepsilon_{1,2}
\end{gathered}
$$

je nachdem, ob $z \leqq h$. $\left(\varepsilon_{2}<\varepsilon_{1}\right)$. Wir setzen

$$
k_{1}^{2}-k_{2}^{2}=\Delta(>0) \text {. }
$$

Neben der Erfüllung der Differentialgleichung (2) unterliegen die Funktionen $\Pi_{1,2}$ noch den Bedingungen:

a) für $z=0$ und $z=h$ müssen die Tangentialkomponenten von $E$ und $H$ stetig verlaufen, was sich in $\Pi$ folgendermaßen schreibt:

$$
\begin{aligned}
& \text { in } z \leq 0: \Pi_{1}=0 ; \\
& \text { in } z=h: \Pi_{1}=I_{2}, \quad \frac{\partial \Pi_{1}}{\partial z}=\frac{\partial \Pi_{2}}{\partial z} .
\end{aligned}
$$

b) Im Sender, $r=0, z=\zeta$, wird $\Pi$ unendlich, wie $e^{i k R} / R$, wo $R$ der vom Sender aus gezogene Kugelradius ist.

c) Im Unendlichen unterliegt unsere Lösung der Ausstrahlungsbedingung.

\section{Integraldarstellung der Lösung}

Man hat für die primäre Erregung $e^{i k R} / R$ die folgende bekannte Integraldarstellung:

$$
\frac{e^{i k R}}{R}=\left\{\begin{array}{l}
\int_{0}^{\infty} J_{0}(\lambda r) \frac{\exp \left[-\sqrt{\left.\lambda^{2}-k_{1}{ }^{2}(z-\zeta)\right] \lambda d \lambda} z>\zeta\right.}{\sqrt{\lambda^{2}-k_{1}{ }^{2}}} z>{ }_{0}^{\infty} \int_{0}^{\infty} J_{0}(\lambda r) \frac{\exp \left[+\sqrt{\left.\lambda^{2}-k_{1}{ }^{2}(z-\zeta)\right] \lambda d \lambda}\right.}{\sqrt{\lambda^{2}-k_{1}{ }^{2}}} z<\zeta,
\end{array}\right.
$$

wobei bekanntlich das Vorzeichen der Wurzel so bestimmt ist, daß für $\lambda \rightarrow+\infty \sqrt{\lambda^{2}-k_{1,2}{ }^{2}} \rightarrow \lambda$.

Mit

schreiben wir:

$$
\begin{aligned}
& \tau_{1}=-i \sqrt{\lambda^{2}-k_{1}{ }^{2}} \\
& \tau_{2}=-i \sqrt{\lambda^{2}-k_{2}{ }^{2}}
\end{aligned}
$$

a) im oberen Medium $z>h$

$$
\Pi=\Pi_{2}=\int_{0}^{\infty} J_{0}(\lambda r r) e^{-i \tau_{2} z f_{3}(\lambda)} j i \tau_{2} \lambda
$$

b) im oberen Teil des unteren Raumes $h>z>\zeta$

$$
\Pi=\Pi_{1 \mathrm{o}}=\int_{0}^{\infty} J_{0}(\lambda r) \frac{\lambda}{j \tau_{1}}\left[e^{+i \tau_{1}(\zeta-z)}+e^{+i \tau_{1} z} f_{1}(\dot{\lambda})+e^{-i \tau_{1} z} f_{2}(\lambda)\right] d i
$$

c) im unteren Teil des unteren Raumes $\zeta>z>0$

$$
\Pi=\Pi_{1 \mathrm{u}}=\int_{0}^{\infty} J_{0}(\lambda r) \frac{\lambda}{j \tau_{1}}\left[e^{i \tau_{1}(z-\xi)}+e^{i \tau_{1} z} f_{1}(\lambda)+e^{-i \tau_{1} z} f_{2}(\lambda)\right] d \lambda .
$$

Man bestimmt nun die Funktionen $f_{1}(\lambda), f_{2}(\lambda), f_{3}(\lambda)$ so, daß die Integrale (8), (9), (10) die oben formulierten Grenzbedingungen in $z=0$ und $z=h$ erfüllen und erhält dann die folgenden Lösungen:

$\operatorname{In} z=h$

In $h>z>\zeta$

$$
I I_{2}=\int_{0}^{\infty} J_{0}(\lambda r r) \frac{2 e^{-i \tau_{2} z}\left(e^{+i \tau_{1} \xi}-e^{-i \tau_{1} \xi}\right)}{j\left(\tau_{1}+\tau_{2}\right) \cdot\left(1+C_{r} e^{-2 i \tau_{1} h}\right)} \lambda d i
$$

In $\zeta>z>0$

$$
I_{1 \mathrm{o}}=\int_{0}^{\infty} J_{0}(\lambda r) \frac{\left(e^{i \tau_{1} \xi}-e^{-i \tau_{1} \xi}\right)\left(e^{-i \tau_{1} z}+C_{r} e^{j \tau_{1}(z-2 h)}\right)}{j \tau_{1}\left(1+C_{r} e^{-2 j \tau_{1} h}\right)} \lambda d \lambda
$$

$$
\Pi_{1 \mathrm{u}}=\int_{0}^{\infty} J_{0}(\lambda r) \frac{\left(e^{j \tau_{1} z}-e^{-i \tau_{1} z}\right)\left(e^{-i \tau_{1} \xi}+C_{r} e^{j \tau_{1}(\xi-2 h)}\right)}{i \tau_{1}\left(1+C_{r} e^{-2 i \tau_{1} h}\right)} \lambda d \lambda
$$


$\Pi_{1}$ o und $\Pi_{1}$ u gehen auseinander durch Vertauschen von $z$ und $\zeta$ hervor; (13) zeigt das Verschwinden von $I_{1 \text { u }}$ in $z=0$.

Es bedeutet dabei

$$
C_{r}=\left(\tau_{1}-\tau_{2}\right) /\left(\tau_{1}+\tau_{2}\right) .
$$

Aus dem physikalischen Teil ergibt sich, daß dies der Koeffizient der Fresnelschen Reflexion ist.

\section{Diskussion der Integrale}

A) Die Singularitäten und Verzweigungen der Integranden

1. Wir haben zunächst in sämtlichen Integranden 2 Verzweigungspunkte: $\lambda=k_{1}$ und $\lambda=k_{2}$. Für die in sie einmündenden Schnitte fassen wir die folgen-

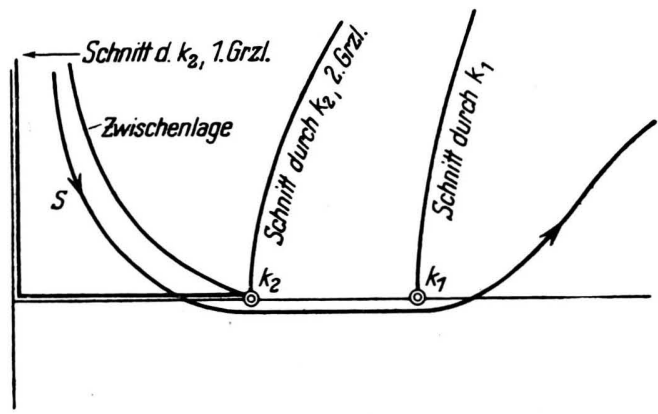

Abb. 3. Die Verzweigungsschnitte und der Integrationsweg in der $\lambda$-Ebene.

den Grenzlagen und alle möglichen Zwischenlagen ins Auge (NB! $k_{1,2}$ rein positiv reell) (Abb. 3).

a) Der Schnitt durch $k_{2}$ :

1. Grenzlage: Vom positiv Unendlichen der imaginären Achse nach Null, längs der positiv reellen Achse nach $k_{2}$.

2. Grenzlage: Vom Punkt $k_{2}$ senkrecht nach oben längs einer gleichseitigen Hyperbel, die nach rechts oben abbiegt, und deren Asymptote die Halbierende des 1. Quadranten ist.

b) Der Schnitt durch $k_{1}$ : Eine solche Hyperbel, vom Punkte $\lambda=k_{1}$ nach oben gehend und nach rechts oben abbiegend.

2. In der so aufgeschnittenen $\lambda$-Ebene suchen wir die Nullstellen des Nenners. Eine gründlichere Untersuchung, die hier $\mathrm{zu}$ weit führen würde ${ }^{3,4}$, zeigt:

1 T. K a h a n u. G. E ckart, C. R. hebd. Séances Acad. Sci. 228, 235 [1949].

2 G. E cka rt u. T. K a h a n, C. R. hebd. Séances Acad. Sci. 228, 304 [1949].

3 G. E ck art u. T. K a h a n, Rev. sci. 86, 723 [1950]. a) Der Nenner der Integrale hat eine Nullstelle bei $\lambda=k_{1}$; dort hat aber auch der Zähler eine Nullstelle der gleichen Ordnung, so daß dieser Punkt keinen Pol darstellt.

b) Schneiden wir nach der 1. Grenzlage auf, so sind Nullstellen des Nenners im oberen Blatt der $\lambda$-Fläche nur möglich auf dem Stück der reellen $\lambda$-Achse zwischen $k_{1}$ und $k_{2}$, und zwar hat man keine Nullstelle, solange

$$
/ \Delta h<\pi / 2 .
$$

Man hat eine Nullstelle, wenn

$$
\pi / 2<\sqrt{\Delta} h<3 \pi / 2 .
$$

Man hat zwei Nullstellen, wenn:

$$
3 \pi / 2<\sqrt{\Delta} h<5 \pi / 2 .
$$

Wenn $\sqrt{\Delta} h$ zunimmt, wächst die Zahl der Nullstellen unbegrenzt ${ }^{3,4}$. Die Nullstellen treten bei $\lambda=k_{2}$ in das obere Blatt unserer Riemannschen Fläche ein und laufen dann gegen $k_{1}$, ohne diesen Punkt jemals zu erreichen. In der unteren Hälfte der rechten $\lambda$-Halbebene gibt es keine Pole.

3. Integrationswege. Unser Integrationsweg war bisher die reelle Achse, wenn nötig unter Umgehung der dort befindlichen Singularitäten durch kleine Kreisbögen nach unten. Jetzt schreiben wir

$$
J_{0}(\lambda r)=\frac{1}{2}\left[\mathfrak{h}_{0}{ }^{(1)}(\lambda r)+\mathfrak{h}_{0}{ }^{(2)}(\lambda r)\right]
$$

und weiter die Integrale (11), (12), (13) in der verkürzten Form:

$$
\begin{aligned}
\Pi=\frac{1}{2} \int_{0}^{\infty} \mathfrak{h}_{0}{ }^{(1)}(\lambda r r) f\left(\tau_{1}, \tau_{2}\right) \lambda d \lambda \\
+\frac{1}{2} \int_{0}^{\infty} \mathfrak{h}_{0}{ }^{(2)}(\lambda r) f\left(\tau_{1}, \tau_{2}\right) \lambda d \lambda
\end{aligned}
$$

wobei wir noch darauf hinweisen, daß in diesen Integralen die Integranden ungerade Funktionen von $\lambda$ sind: $\lambda$ tritt in $\tau_{1.2}$ nur quadratisch auf. $J_{0}(\lambda r)$ ist eine gerade Funktion von $\lambda$, so daß der Faktor $\lambda$ hiefür bestimmend ist.

Wir verformen den Integrationsweg jetzt in bekannter Weise: Den Weg des Integrals mit $\mathfrak{S}_{0}{ }^{(2)}$ ziehen wir an die negative imaginäre Achse heran.

$4 \mathrm{~T} . \mathrm{Kahan} \mathrm{u}$ G. Eckart, Théorie du Guide d'Onde Atmosphérique. Forschungsbericht von Office National d'Etudes et de Recherches Aéronautiques, Paris No. 1029 P. I, Note technique No. 1 [1949].

5 T. K a h a n u. G. E c k a rt, J. Physique Radium (VIII) 10, 165 [1949]. 
Den Weg des Integrals mit $\mathfrak{S}_{0}{ }^{(1)}$ verlagern wir nach oben, so daß er an die positiv imaginäre Achse herankommt; dann folgt er von oben im Unendlichen kommend dem Schnitt durch $k_{2}$, verläuft dann längs der reellen Achse nach $k_{1}$ und geht dann nach oben ins Unendliche in dem Raum zwischen der positiv reellen $\lambda$-Achse und dem Hyperbelschnitt durch $k_{1}$. Infolge der bekannten asymptotischen Eigenschaften der Hankelschen Funktionen und der Halbumlaufsrelationen der Zylinderfunktionen verschwinden die Inte- grale über die unendlich fernen Kreisbögen, und die Integrale, die vom Nullpunkt aus nach oben und unten den imaginären Halbachsen folgen, heben sich gegenseitig weg (Abb.3).

B) Wellenleiter geringer Höhe oder kurze Wellen

Unter der Voraussetzung $\sqrt{\Delta h}<\pi / 2$ haben wir dann folgendes: Zunächst nehmen die Integrale die Gestalt an:

$$
\begin{aligned}
& I I_{2}=\frac{1}{2} \int_{s} 2 \mathfrak{h}_{0}{ }^{(1)}\left(\lambda_{r} r\right)\left\{\frac{e^{-i \tau_{2} z}}{j\left(\tau_{1}+\tau_{2}\right)} e^{j\left(\tau_{2}-\tau_{1}\right) h}\left(\begin{array}{c}
\left(\tau^{i \tau_{1}} \xi\right. \\
1+C_{r} e^{-2 i \tau_{1} h}
\end{array}\right\} \lambda d \lambda,\right. \\
& \Pi_{1 \mathrm{o}}=\frac{1}{2} \int_{s} \mathfrak{T}_{0}{ }^{(1)}(\lambda r)\left\{\frac{\left(e^{i \tau_{1} \zeta}-e^{-i \tau_{1} \zeta}\right)\left[e^{-i \tau_{1} z}+C_{r} e^{j \tau_{1}(z-2 h)}\right]}{j \tau_{1}\left(1+C_{r} e^{-2 i \tau_{1} h}\right)}\right\} \lambda d \lambda, \\
& \Pi_{1 \mathrm{u}}=\frac{1}{2} \int_{s} \mathfrak{h}_{0}{ }^{(1)}(\lambda r r)\left\{\frac{\left(e^{i \tau_{1} z}-e^{-i \tau_{1} z}\right)\left[e^{-i \tau_{1} \xi}+C_{r} e^{j \tau_{1}(\xi-2 h)}\right]}{j \tau_{1}\left(1+C_{r} e^{-2 i \tau_{1} h}\right)}\right\} \lambda d i,
\end{aligned}
$$

wo $s$ den oben angegebenen Integrationsweg darstellt. Man diskutiert diese Integrale asymptotisch ${ }^{4}$ nach der Sattelpunktsmethode. Das Ergebnis ist folgendes: Unter einigermaßen steilen Erhebungswinkeln ergibt sich für das Feld im Raume oberhalb des Ducts:

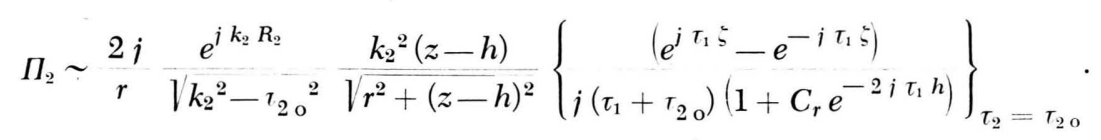

$\tau_{20}$ bedeutet dabei den Sattelpunkt, definiert durch

$$
\tau_{20}=-\frac{k_{2}(z-h)}{\mid \sqrt{r^{2}+(z-h)^{2}}}, z>h .
$$

Dadurch ist $\lambda$ festgelegt und daher auch $\tau_{1}$ vermittels (6) und (7), somit die geschweifte Klammer in (21) für $\tau_{2}=\tau_{20}$.

Unter kleineren Erhebungswinkeln unmittelbar oberhalb der Trennfläche erhält man:

$$
\Pi_{2} \sim \frac{1}{r^{3}} e^{-\sqrt{\Delta}(z-h)}
$$

Im Wellenleiter selbst hat man unter Voraussetzung $\sqrt{\Delta} h<\pi / 2$, also im Falle des niedrigen Ducts oder der längeren Wellen, das Ergebnis, daß asymptotisch das Feld mit $1 / r^{3}$ abnimmt. Wenn der Sprung von $\varepsilon$ in der Höhe $h$ nicht vorhanden wäre, würde man erhalten

$$
\Pi_{2}=-2 j k_{1} e^{i k_{1} r} z \zeta / r^{2} .
$$

Dadurch, daß aber nun noch die Reflexion in der Höhe $h$ hinzukommt, löscht sich das Feld der Ordnung $1 / r^{2}$ aus und es bleibt nur ein Feld der Ordnung $1 / r^{3}$.

C) Fall des höheren Ducts, Fall der kürzeren Wellen

Ist der Wellenleiter höher oder die Welle kürzer, so haben wir folgende Verhältnisse. Betrachten wir zunächst das :Feld unterhalb der Trennfläche! Hier haben wir Pole des Integranden auf der reellen Achse zwischen $k_{2}$ und $k_{1}$. Wie eine tiefere Untersuchung ${ }^{3,4}$ zeigt, existiert für

$$
\begin{array}{rlrl}
0 & <2 \sqrt{\Delta} h<\pi & & \text { kein Pol, } \\
\pi & <2 \sqrt{\Delta} h<3 \pi & & 1 \text { Pol }, \\
3 \pi & <2 \sqrt{\Delta} h<5 \pi & 2 \text { Pole }
\end{array}
$$$$
(2 n-1) \pi<2 \sqrt{\Delta} h<(2 n+1) \pi \quad n \text { Pole } .
$$ 


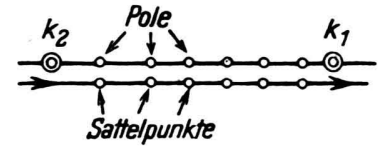

Abb. 4. Die Pole und die sich darunter ausbildenden Sattelpunkte.

Wir brauchen nunmehr den Integrationsweg nicht über diese Pole hinwegzuziehen, sondern können direkt ihren asymptotischen Einfluß auf das Integral erkennen (Abb.4), wenn wir uns überlegen, daß sich asymptotisch unter jedem Pol ein Sattelpunkt ausbildet, dessen Beitrag zum Integral gerade die asympto- tische Darstellung des Residuums ist. Es ist dies der analoge Fall zu dem Sattelpunkt, den die Verfasser in der Theorie der Oberflächenwelle angegeben haben und der im Verzweigungsschnittintegral den Anteil liefert, der das Residuum, die Oberflächenwelle auskompensiert ${ }^{6,7,8}$.

Wir bezeichnen nun die $\lambda$-Werte, die den Polen zugeordnet sind, mit dem laufenden Index $s$ als $\lambda_{\mathrm{s}}$, die Anteile, die diese Pole zu $\Pi_{2}, \Pi_{10}, \Pi_{1 \text { u liefern }}$ als $P_{2}^{(n)}, P_{1 \mathrm{o}}^{(n)}, P_{1 \mathrm{u}}^{(n)}$, wobei der obere Index $n$ die Summation über $n$ Pole $(s=1 \ldots n)$ andeuten soll, und erhalten, wenn wir die Hankel-Funktion asymptotisch einsetzen:

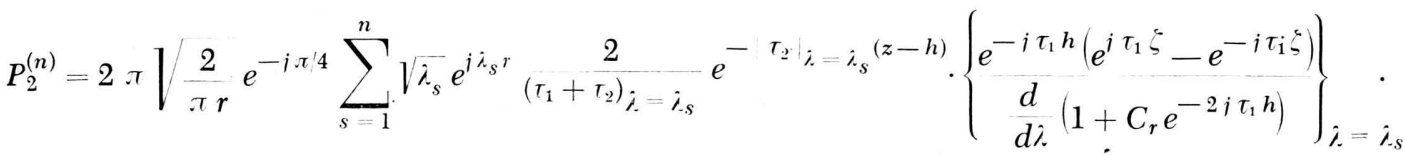

$$
\begin{aligned}
& P_{1 \mathrm{o}}^{(n)}=2 \pi \sqrt{\frac{2}{\pi r} e^{-i \pi / 4} \sum_{s=1}^{n} \sqrt{\lambda_{s}} e^{i \lambda_{s} r}{ }_{\left(\tau_{1}\right) j_{z}=\lambda_{s}}^{1}} \cdot\left\{\frac{\left(e^{i \tau_{1} \zeta}-e^{-i \tau_{1} \zeta}\right)\left[e^{-i \tau_{1} z}+C_{r} e^{i \tau_{1}(z-\Sigma h)}\right]}{\frac{d}{d i}\left(1+C_{r} e^{-2 i \tau_{1} h}\right)}\right\}_{\lambda=i s s} . \\
& P_{1 \mathrm{u}}^{(n)}=2 \pi \sqrt{\frac{2}{\pi r}} e^{-i \pi / 2} \sum_{s=1}^{n} \sqrt{\lambda_{s}} \frac{e^{i \lambda_{s} r}}{\left(\tau_{1}\right)_{\lambda=\lambda_{s}}} \cdot\left\{\begin{array}{c}
\left(e^{i \tau_{1} z}-e^{\left.-i \tau_{1} z\right)\left(e^{-i \tau_{1} \xi}+C_{r} e^{+i \tau_{1}(\xi-2 h)}\right)}\right. \\
\frac{d}{d \lambda}\left(1+C_{r} e^{\left.-2 i \tau_{1} h\right)}\right.
\end{array}\right\}_{\lambda=\lambda_{s}} .
\end{aligned}
$$

Man sieht aus (25), daß das Feld dieser Wellen von der Trennfläche aus nach oben zu exponentiell abnimmt. In Abhängigkeit vom Zylinderradius $r$ nehmen die Wellen mit $1 / \sqrt{r}$ ab. Innerhalb des Ducts ist über die Abhängigkeit von $z$ folgendes zu sagen: Für $z=0$ ist $\Pi$ Null und wächst mit wachsendem $z$ nach einem Sinusgesetz, also zunächst linear an. Es sind Hohlleiterwellen, auf die wir im physikalischen Teil noch zurückkommen werden.

D) Das Gebiet bis zum Einsetzen der Totalreflexion

1. Die bisherigen Betrachtungen waren asymptotischer Natur, d.h. gültig in genügend großen Entfernungen. Sie ergaben in dem Wellenleiter Abnahmegesetze für die Feldstärke nach $1 / r^{3}$ und $1 / V / r$ ent-

6 T. K a ha n u. G. E ck a r t, J. Physique Radium (VIII) 10, 333 [1949].

7 T. Ka han u. G. E ckart, Physic. Rev. 76, 406 [1949].

8 T. K a han u. G. E cka rt, C. R. hebd. Séances Acad. Sci. 227, 969 [1948]. sprechend der Frequenz und der Höhe des Ducts. Andererseits haben wir zunächst um den Sender herum sichèr ein Gesetz der Ordnung 1/R, und wir wollen jetzt sehen, wie weit der Gültigkeitsbereich sich erstreckt.

Der Nenner von (18), (19), (20) legt folgende Entwicklung nahe:

$$
\frac{1}{1+C_{r} e^{-2 i \tau_{1} h}}=\sum_{\nu=}^{\infty}(-1)^{\nu}\left(C_{r} e^{-2 j \tau_{1} h}\right)^{\nu} .
$$

Man kann zeigen ${ }^{4}$, daß man diese Entwicklung anwenden kann, solange der Integrationsweg das Stück zwischen $k_{1}$ und $k_{2}$ auf der reellen $\lambda$-Achse oder das Stück zwischen $-\sqrt{\Delta}$ und Null auf der $\tau_{1}$-Achse vermeidet.

Treiben wir die Entwicklung (28) etwa bis zum $n$-ten Glied und schließen wir mit einem Restglied $R_{n}$ ab, können wir $\Pi_{1}$ o folgendermaßen schreiben, wobei wir die Hankelsche Funktion durch ihre asymptotische Darstellung ersetzen: 
$\Pi_{10}=\sqrt{\frac{2}{\pi r}} e^{i \pi / 4} \int_{s} \frac{e^{i \lambda r}}{\sqrt{r}} \frac{\left(e^{i \tau_{1} \zeta}-e^{-i \tau_{1} \zeta}\right)}{j \tau_{1}}\left[e^{i \tau_{1} z}+C_{r} e^{j \tau_{1}(z-2 h)}\right] \cdot\left\{\sum_{v=0}^{n} C_{r}^{v}(-1)^{v} e^{-2 i v \tau_{1} h}+R_{n}\right\} \lambda d i$.

2. Wenn man den Integrationsweg so führt, daß auf ihm die Entwicklung kcnvergiert, kann man gliedweise integrieren und erhält schließlich folgenden Ausdruck:

$$
\begin{aligned}
& \Pi_{10}=-i \sqrt{r_{\pi}} e^{-i \pi / 4}\left\{\int_{s} \frac{e^{i j r}}{\sqrt{\lambda}} \frac{\left(e^{i \tau_{1} \zeta}-e^{-i \tau_{1} \xi}\right)}{\tau_{1}} e^{-i \tau_{1} z} \lambda d \lambda+\int_{s} \frac{e^{i \lambda r}}{V^{\lambda}} \frac{\left(e^{i \tau_{1} \zeta}-e^{-i \tau_{1} \zeta}\right)}{\tau_{1}} C_{r} e^{-i \tau_{1}(2 h-z)} \lambda d i\right. \\
& +\int_{s} \frac{e^{i \lambda_{r}}}{\sqrt{\lambda}} \frac{\left(e^{i \tau_{1} \zeta}-e^{-i \tau_{1} \zeta}\right)}{\tau_{1}}\left(-C_{r}\right) e^{-i \tau_{1}(2 h+z)} i d \lambda+\int_{s} \frac{e^{i \lambda_{r}}}{l_{i}}\left(e^{i \tau_{1} \zeta}-e^{\left.-i \tau_{1} \zeta\right)}\left(-C_{r}{ }^{2}\right) e^{-i \tau_{1}(2 \cdot 2 h-z)} \dot{\lambda} d i\right. \\
& +\int_{s} \frac{e^{i \lambda r}}{\sqrt{\lambda}} \frac{\left(e^{i \tau_{1} \zeta}-e^{-i \tau_{1} \zeta}\right)}{\tau_{1}}\left(C_{r}\right)^{2} e^{-i \tau_{1}(2 \cdot 2 h+z)} i d i+\int_{s} \frac{e^{i \lambda_{r} r}}{l i} \frac{\left(e^{i \tau_{1} \zeta}-e^{-i \tau_{1} \zeta}\right)}{\tau_{1}}\left(C_{r}\right)^{3} e^{-i \tau_{1}(3 \cdot 2 h-z)} i d \dot{ }
\end{aligned}
$$

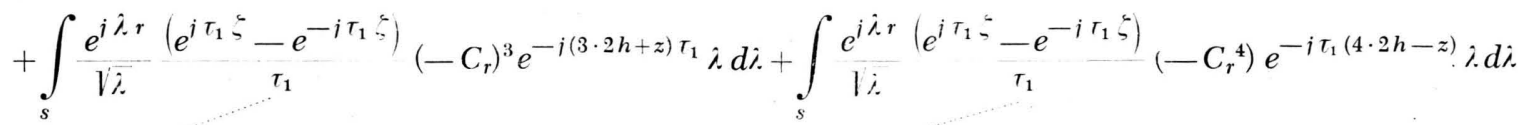

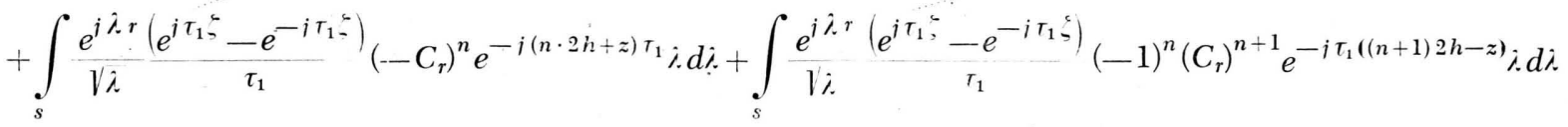

$$
\begin{aligned}
& +\int_{s} \frac{e^{i \lambda r}\left(e^{i \tau_{1} \zeta}-e^{-i \tau_{1} \zeta}\right)}{\tau_{1}} e^{-2 j(n+1) \tau_{1} h}(-1)^{n+1}\left(C_{r}\right)^{n+1} e^{-j \tau_{1} z}+C_{r} e^{j \tau_{1}(z-2 h)}\left(1+C_{r} e^{\left.-2 j \tau_{1} h\right)} \quad \lambda d \lambda\right\}
\end{aligned}
$$

Das erste Glied dieser Entwicklung stellt einfach die vom Sender und seinem Spiegelbild im Boden ausgehende primäre Welle dar.

Die anderen Integrale kann man nach der Sattelrunktsmethode behandeln. Wir suchen nun die Bereiche in $r$, wo die Sattelpunkte, die ja die Hauptanteile der Integrale liefern, im Konvergenzgebiet von (28) liegen. Dies ist der Fall, wenn

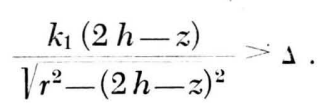

Der Sinn dieser Bedingung soll im physikalischen Teil behandelt werden. In diesem Bereich führt (30) auf eine Ausbreitung nach $1 / R$, die im physikalischen Teil ebenfalls erläutert werden soll.

E) Bemerkung über $I I$ und die Feldstärke $\mathfrak{g}$ und $E$

Wir haben hier ausschließlich das Fitzgeraldsche Vektorpotential $\Pi$ berechnet. Der Fitzgeraldsche Vektor ist nun das magnetische Analogon zum Hertzschen Vektor. Man hat im Fernfeld des Hertzschen Dipols nur $E$ und $\mathfrak{g}$ zu vertauschen, um zum Felde des magnetischen Dipols zu gelangen. Wenn wir uns hier auf das magnetische Feld $H$ beschränken, das in der Meridianebene senkrecht zum Radiusvektor liegt, oder auf das in Kreisen um die $z$-Achse liegende elektrische Feld, so haben wir hier in der primären Strahlung ein Vertikaldiagramm der Form $\sin \vartheta$ (Abb. 5). Die Verteilung des Hertzschen und des Fitzgeraldschen Vektors ist dagegen gleichmäßig im Raum (unabhängig vom Erhebungswinkel). Unter flachen Erhebungswinkeln kann man $\mathfrak{S}$ und $E$ als proportional $\Pi$ ansehen, während bei Winkeln um $90^{\circ} \Pi$ noch mit $\sin \vartheta=\cos \alpha(\alpha=\pi / 2-\vartheta) \mathrm{zu}$ multiplizieren ist (Abb.6).

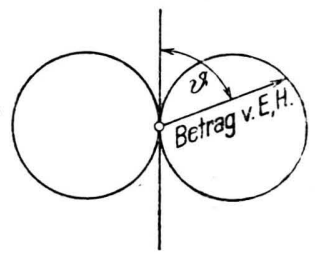

Abb. 5

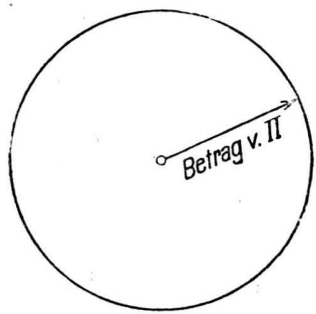

Abb. 6
Abb. 5. Vertikaldiagramm der Feldstärkenverteilung eines Dipols.

Abb. 6. Vertikaldiagramm des Hertzschen und Fitzgeraldschen Vektors eines Dipols. 


\section{Physikalischer Teil}

1. Physikalische Bedeutung der Größen $\lambda, \tau_{1}, \tau_{2}$

Um den physikalischen Sinn der obigen Formeln aufzuhellen, müssen wir zuerst die Bedeutung der Größen $\lambda, \tau_{1}, \tau_{2}$ angeben. Es ist

$$
\lambda=k_{1} \sin \vartheta_{1}
$$

wenn $\vartheta_{1}$ der Richtungswinkel der Wellennormalen gegen die $z$-Achse ist (im unteren Medium). Damit ist aber auch (6), (7)

oder

$$
\tau_{1}^{2}=k_{1}^{2}\left(1-\sin ^{2} \vartheta_{1}\right)
$$

$$
\tau_{1}=k_{1} \cos \vartheta_{1} .
$$

Analog ist im oberen Medium

und daher

$$
\lambda=k_{2} \sin \vartheta_{2}
$$

$$
\tau_{2}=k_{2} \cos \vartheta_{2} .
$$

Man sieht aus (34) und (35) direkt das Fresnelsche Brechungsgesetz

$$
\lambda=k_{1} \sin \vartheta_{1}=k_{2} \sin \vartheta_{2} .
$$

Wenn wir dann in einer der obigen Integralformeln in einem Integranden der Form $e^{i \lambda r} f\left(r, \tau_{1}, \tau_{\mathbf{2}}\right)$ die einem Sattelpunkt entsprechenden Werte einsetzen, so haben wir damit bereits in dem Wellenbündel die dominierenden Teile hervorgehoben, die Teile des Bündels, die nach Größe und Richtung den physikalischen Vorgang in erster Näherung bestimmen. Dies ist in unserem Falle die physikalische Bedeutung der Sattelpunktsmethode, und von diesem Gesichtspunkt aus wollen wir jetzt unsere Formeln diskutieren.

\section{Das Vertikaldiagramm des magnetischen Dipols}

Wir betrachten zunächst den Faktor $e^{j \tau_{1} \xi}-e^{-j \tau_{1} \xi}$, der in vielen Formeln auftritt und auf den wir schon im mathematischen Teil hingewiesen haben. Er stellt, wenn man $\tau_{1}=k_{1} \cos \vartheta_{1}$, also $\vartheta$ verschiedene Werte durchlaufen läßt, das Vertikaldiagramm eines in der Höhe $\zeta$ befindlichen magnetischen Dipols dar, das aus einer Reihe von Lappen besteht, je nachdem wie groß die Wellenlänge und die Höhe $\zeta$ ist. Diese Lappen rühren bekanntlich vom Gangunterschied eines Strahles her, der vom Sender ausgeht, und des Strahles, der von seinem gegenphasigen Spiegelbild ausgeht. In $\Pi$ geschrieben sind die Lappen alle von derselben Größe, in $H$ oder $E$ geschrieben hat das Diagramm jedoch die halbkreisförmige Begrenzungslinie, auf die wir am Schluß des mathematischen Teils hinwiesen (Abb. 5, 6, 7).

3. Die Verhältnisse im oberen Raum in größeren Entfernungen (Abb. 8)

Wir haben gesehen, daß, solange $z-h$ in der Größenordnung von $r$ ist, sich das ursprüngliche

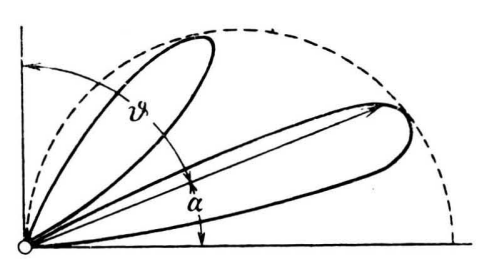

Abb. 7

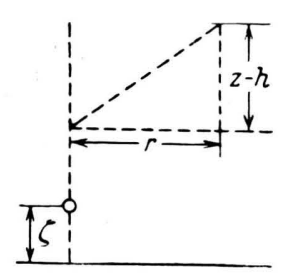

Abb. 8
Abb. 7. Lappendiagramm im Halbkreis.

Abb. 8. Koordinaten oberhalb der Trennfläche.

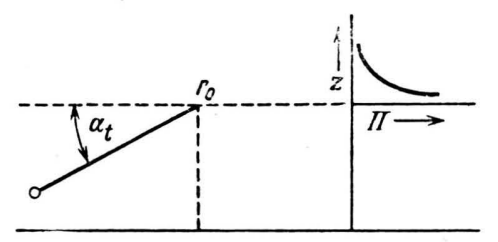

Abb. 9. Totalreflexionswinkel und Verteilung von $\Pi$ unmittelbar über der Trennfläche.

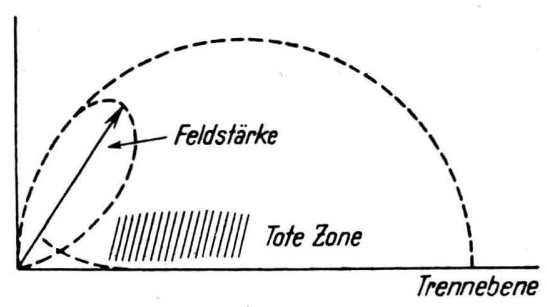

Abb. 10. Tote Zone.

Diagramm mit einem kleinen Knick (Brechung!) nach oben fortpflanzt. Der Sattelpunkt liegt, wie man leicht zeigt ${ }^{4}$, in der $\tau_{2}$-Ebene bei

$$
\tau_{20}=\frac{k_{2}(z-h)}{\sqrt{r^{2}+(z-h)^{2}}} .
$$

Oder: man hat mit Rücksicht auf (21) in $1 / r$ ein Feld, das wegen des Faktors $(z-h)$ gegen flache Erhebungswinkel zu stark abnimmt.

In der unmittelbaren Nachbarschaft der Trennebene ergibt sich aus den Formeln (23) und (25), daß das Feld, das im Duct vorhanden ist, sich nach oben 
zu oberhalb desselben mit einer exponentiellen Dämpfung fortsetzt, solange $r$ groß genug ist. Dies ist eine Erscheinung, die von der Totalreflexion her bekannt ist. Wir zeichnen in der Figur rechts die zAchse in vertikaler Richtung und senkrecht dazu, horizontal, oberhalb des Ducts den Betrag des Vektors $\Pi$, der exponentiell abnimmt. Man erhält so (Abb. 9, 10) unabhängig von der Lappenbildung in 5 und $E$, die in $e^{j \tau_{1} \zeta}-e^{-j \tau_{1} \zeta}$ enthalten ist, ein Vertikaldiagramm, das für kleine Erhebungswinkel oberhalb des Ducts die bekannte tote Zone liefert. Dies gilt natürlich nur, wenn die Entfernung so groß ist, daß sich an der Trennfläche Totalreflexion ausbildet.

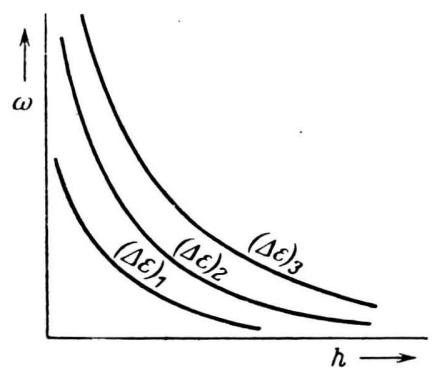

Abb. 11. Grenzfrequenz als Funktion der Ducthöhe, $\Delta \varepsilon$ Parameter.

\section{Das Feld im Duct}

Wir hatten aus Gl. (23) gesehen, daß im, ,niedrigen“ Duct das Feld mit $1 / r^{3}$ gegen Null geht. Wir werden den Ausdruck „niedriger“ Duct noch etwas präzisieren: Die Grenze zwischen niedrigen und höheren Ducts ist gegeben durch die Gleichung (15a)

$$
\omega \sqrt{\varepsilon_{0} \mu_{0}} u\left(\varepsilon_{1}-\varepsilon_{2}\right) h=\pi / 2 .
$$

Ist $h$ oder $\omega$ größer, als dieser Bedingung entspricht, sind im oberen Blatt der Riemannschen $\lambda$-Fläche Pole vorhanden, die zum Auftreten von ungedämpften Hohlleiterwellen der Ordnung $1 / / r$ Veranlassung geben. Ist $h$ kleiner, $\omega$ kleiner und $\lambda$ größer, als dieser Bedingung entspricht, sind diese Wellen exponentiell gedämpft, und es dominiert der Term mit $1 / r^{3}$. Somit ist durch (15a) die Grenze zwischen niedrigem und hohem Duct, zwischen kürzeren und längeren Wellen wohl definiert. Wir geben dieser Bedingung in der Abb. 11 eine kurvenmäßige Darstellung. Wir zeichnen für verschiedene Werte von $\Delta \varepsilon=\varepsilon_{1}-\varepsilon_{2}$ als Parameter $\omega$ als Funktion von $h$, so, daß es der obigen Bedingung (15a) entspricht. Dann entsprechen die jeweils weiter unten liegenden Kurven größeren Werten von $\Delta \varepsilon$. Ist für ein festes $\Delta \varepsilon \omega$ oberhalb der durch diese Kurven gegebenen Grenze, so hat man ein Ausbreitungsgesetz der Form $1 / / r$, wenn man weit genug in den Duct hinein geht. Das heißt aber, daß die Energie, die unter dem Winkel der Totalreflexion in den Duct eindringt, sich dort in Form einer exponentiell ungedämpften Hohlleiterwelle ausbreitet, die eine Zylinderwelle mit dem Gesetz $1 / / r$ darstellt. Da die Leistung proportional dem Quadrat des Feldes ist, wird die Zylinderwand der Höhe $h$ und vom Radius $r$ in allen Entfernungen von einer konstanten Leistung $2 \pi r h / r$ durchströmt. Diese Hohlleiterwellen haben in unserem Fall die Form von $H$ - oder $T E$-Wellen, denn wir haben nur die Komponente $E_{q}$, die überall normal zur Ausbreitungsrichtung ist, so daß nur eine Komponente von $\mathfrak{b}$ in dieser gelegen sein kann. Wenn $h$ oder $\omega$ oder $\Delta \varepsilon$ zu klein sind, können solche Wellen nicht entstehen, ohne daß sie exponentiell gedämpft sind und müssen gegenüber dem Glied mit $1 / r^{3}$ zurücktreten. Das heißt aber, daß der Duct die längeren Wellen sperrt.

\section{Entwicklung nach Reflexionen und ihr Gültigkeitsbereich}

Wir haben bis jetzt die sehr großen Entfernungen diskutiert. Im mathematischen Teil haben wir die Zone zwischen dem Sender und diesem entfernten Gebiet betrachtet und wollen hier den physikalischen Sinn des dortigen Formelapparats entwickeln. Zunächst ersetzen wir den Sender und sein Spiegelbild am Boden durch einen am Boden befindlichen Sender doppelter Intensität und der Richtcharakteristik nach Abb. $7\left(e^{j \tau_{1} \zeta}-e^{-j \tau_{1} \zeta}\right)$.

Wir können nun das Strahlungsfeld darstellen durch Superposition dieser primären Senderstrahlung und derjenigen ihres an der Trennschicht gebildeten Spiegelbildes. Dies liefert eine Quelle der Intensität $C_{r}$ in der Höhe $2 h$. Dieses Spiegelbild wird seinerseits am Boden reflektiert, und zwar mit dem Zeichen - 1, und stellt einen Sender der Intensität $-C_{r}$ in der Höhe $-2 h$ dar. Wir verstehen hier den Sinn der Gleichung (14) des mathematischen Teils. Der letztere Sender wird wieder an der Schicht in der Höhe $h$ gespiegelt und liefert einen Sender der Intensität $-C_{r^{2}}$ in der Höhe $4 h$. Eine neue Spiegelung am Boden liefert einen Sender der Intensität $+C_{r}{ }^{2}$ in der Höhe $-4 h$. Dies führt zu einem Sender der Intensität $C_{r^{3}}$ in $z=+6 h$, weiterhin zu einem Sen$\operatorname{der}-C_{r}{ }^{3}$ in $z=-6 h$ usw. In $C_{r},-C_{r}, C_{r}^{2},-C_{r}^{2} \ldots$ ist jeweils im $\tau$-Wert dasjenige $\vartheta$ (oder $\lambda$ ) einzusetzen, 
der dem Sattelpunkt des entsprechenden Integrals in (30) entspricht und dabei aus Abb. 12 ersichtlich ist.

Wenn man so verfährt, sieht man, daß für kleine $\varepsilon$-Sprünge $\left|C_{r}\right|=1$ unter sehr flachen Erhebungswinkeln, die im Gebiet der Totalreflexion liegen, und unter etwas steileren Winkeln sehr stark abnimmt. Diese Darstellung nach Reflexionen nach Gl. (30) muß rasch konvergieren, den $C_{r}{ }^{v}(v)$ nimmt mit wachsendem $v$ sehr stark ab.

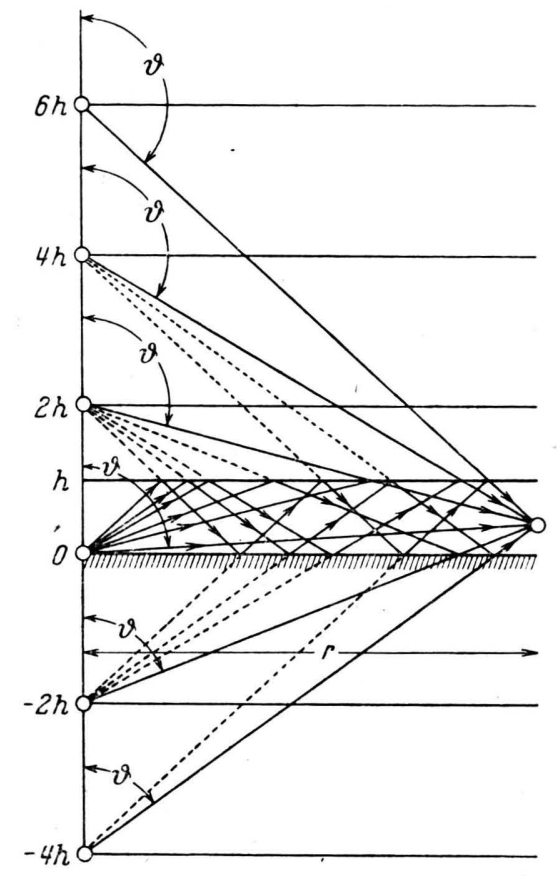

Abb. 12. Die aufeinanderfolgenden Reflexionen. Die verschiedenen Winkel $\vartheta$ sind die Richtungswinkel, die den Sattelpunkten der Integrale in (30) entsprechen.

Die Konvergenzbetrachtungen in ${ }^{4}$ zeigen, daß diese Darstellung gültig bleibt für einen Entfernungsbereich, in dem die erste Spiegelung gerade unter dem Totalreflexionswinkel auftritt. Auf Grund unserer Untersuchungen leuchtet dies nunmehr ein: ein Gesetz der Ordnung $1 / r$ kann nicht mehr gelten, wenn die Entfernung so groß ist, daß ein $1 / / r$-Gesetz gilt. Im streifenden Einfall wird der Reflexionsfaktor an der Schicht mehr und mehr -1 , einfallende und reflektierte Welle löschen sich aus. Eine weitere Spiegelung mit -1 erfolgt am Boden; so versteht man, daß sich auch ein an der Schichtdecke $z=h$ etwa ausbildendes Glied der Ordnung $1 / r^{2}$ zusätzlich weghebt, so daß nur ein Glied mit $1 / r^{3}$ bleibt. So kann man das Glied mit $1 / r^{3}$ als aus Kompensation der $1 / r$ Glieder entstanden denken; ein $1 / \sqrt{r}$-Gesetz kann aber nicht durch eine konvergente Entwicklung der Ordnung $1 / r$ entstehen. Man sieht auch noch folgendes: In dem Bereich unmittelbar hinter dem Einsetzen der Totalreflexion, wo die Quellenentwicklung gilt, werden um so mehr Lappen eingefangen, je kürzer die Welle ist. Man sieht auch hier die Bevorzugung der kürzeren Wellen. Noch ein Wort zum Auftreten der geführten Wellen: Im Falle des niedrigen Ducts liegen die Pole im unteren Blatt der Riemannschen $\lambda$-Fläche in unbegrenzter Anzahl. Ziehen wir den Schnitt durch $k_{2}$ an die 2. Grenzlage

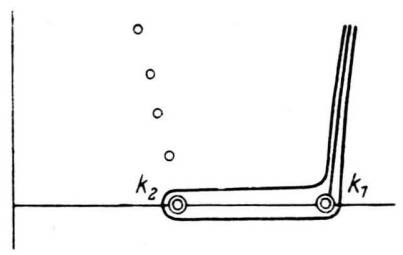

Abb. 13. Komplexe Nullstellen des Nenners in (11), (12), (13).

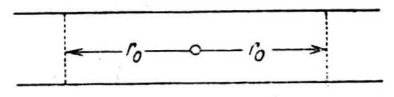

Abb. 14. Dielektrische Platte.

nach rechts (vgl. math. Teil) oder noch weiter nach rechts, etwa in die Linie $k_{2}-k_{1}$, und von dort in den Schnitt durch $k_{1}$ hinein und lassen den Integrationsweg diesem Schnitt folgen (Abb. 13), so überschreiten wir jetzt die zugänglich gewordenen Pole, die in der oberen Halkebene liegen und mit $e^{j \lambda_{s} r}$ eine Amplitudenabnahme nach $e^{-\operatorname{Im}\left(\lambda_{s}\right) r \mid}$ zeigen. Mit zunehmender Höhe des Ducts oder zunehmender Frequenz wandern diese Pole nach $k_{2}$ und von dort auf der reellen Achse nach $k_{1}$, ohne diesen Punkt zu erreichen.

\section{Schlußbemerkung}

Mit unserem Problem haben wir noch ein anderes mitbehandelt, nämlich dasjenige der dielektrischen Flächenantenne (Abb. 14). Nehmen wir die $\varepsilon$-Unstetigkeit größer an, so haben wir damit das Verhalten einer unendlich ausgedehnten dielektrischen Platte studiert. Ein neues Problem würde sich ergeben, wenn man diese Platte in einer Entfernung $r_{0}$ vom Sender abschneidet. 